\title{
ON OBSERVER DESIGN METHODS FOR A CLASS OF TAKAGI-SUGENO FUZZY SYSTEMS
}

\author{
Dusan Krokavec and Anna Filasova \\ Department of Cybernetics and Artificial Intelligence, Technical University of \\ Kosice, Faculty of Electrical Engineering and Informatics, \\ Letna 9, 04200 Kosice, Slovakia
}

\begin{abstract}
The generalized design principle of TS fuzzy observers for one class of continuous-time nonlinear MIMO systems is presented in this paper. The problem addressed can be indicated as a descriptor system approach to TS fuzzy observers design, implying the asymptotic convergence of the state observer error. A new structure of linear matrix inequalities is outlined to possess the observer asymptotic dynamic properties closest to the optimal.
\end{abstract}

\section{KEYWORDS}

Thau observer, TS fuzzy observer, convex optimization, linear matrix inequalities

\section{INTRODUCTION}

As is well known, observer design is a hot research field owing to its particular importance in observer-based control, and fault diagnosis. The nonlinear system theory using Lipchitz conditions has emerged as a method capable of use in state estimation design for nonlinear systems [1], although Lipschitz condition is a restrictive condition which many classes of systems may not satisfy. However this principle used in state estimators design means that the observer satisfies a sufficient condition for the asymptotic stability of error system, but in fact there is not a straightforward method for selecting the observer gain to fill such conditions [2]. Because the Takagi-Sugeno (TS) fuzzy approach is a suitable representation of certain class of nonlinear dynamic systems [3], employing the fuzzy modelling approach to approximate sector-bounded nonlinear systems, other well-known nonlinear observers are based on Takagi-Sugeno (TS) fuzzy models [4], [5]. To design TS fuzzy observers, usually the technique utilizing the linear matrix inequalities is used [6].

Although the state observers for linear and nonlinear systems received considerable attention, the descriptor design principles have not been studied extensively. Adapting the descriptor observer design principle [7], the first result giving sufficient design conditions, but for linear time-delay systems, can be found in [8]. Reflecting the same problems concerning the observers for descriptor time-delay nonlinear systems represented by TS fuzzy models, an LMI method was presented in [9], but a hint of this methodology can be found only in [10].

David C. Wyld et al. (Eds) : SAI, CDKP, ICAITA, NeCoM, SEAS, CMCA, ASUC, Signal - 2014 pp. 279-290, 2014. (C) CS \& IT-CSCP 2014

DOI : $10.5121 /$ csit.2014.41126 
Adapting the results on the TS fuzzy observers for bilinear systems [11] as well as their potential extensions, the main issue of this paper is to use the descriptor principle in TS fuzzy observer design. Preferring LMI formulation, although partly conservative, the stability condition proofs use standard arguments on $\mathrm{H}_{2}$ approach to obtain the design conditions requiring only solving of LMIs without additional constraints. To the best author's knowledge, the proposed LMI structure in design conditions formulation were not fully addressed yet in the previous works.

The paper is organized as follows. In Sec. 2, the TS fuzzy model is briefly described and the TS fuzzy observer design problem for given class of nonlinear systems is formulated in Sec. 3. The new LMI structure, describing the TS fuzzy observer design conditions, is presented in Sec. 4 and analysed and algorithmically explained in Sec 5. Finally, Sec. 6 draws conclusions and some future directions.

The notations throughout the paper are narrowly standard in such a way that $\boldsymbol{x}^{T}, \boldsymbol{X}^{T}$ denotes the transpose of the vector $\boldsymbol{x}$ and matrix $\boldsymbol{X}$, respectively, $\boldsymbol{X}=\boldsymbol{X}^{T}>0$ means that $\boldsymbol{X}$ is a symmetric positive definite matrix, the symbol $\boldsymbol{I}_{n}$ indicates the $n$-th order unit matrix, $R$ denotes the set of real numbers $R^{n}$, and $R^{n \times r}$, refer to the set of all $n$-dimensional real vectors and $n \times r$ real matrices, respectively.

\section{TAKAGI-SUGENO FUZZY MODELS}

The systems under consideration are from the class of multi-input and multi-output nonlinear (MIMO) continuous-time dynamic systems, represented in TS form as

$$
\begin{gathered}
\dot{\boldsymbol{q}}(t)=\sum_{i=1}^{s} \mathrm{~h}_{i}(\boldsymbol{\theta}(t))\left(\boldsymbol{A}_{i} \boldsymbol{q}(t)+\boldsymbol{B}_{i} \boldsymbol{u}(t)\right) \\
y(t)=C q(t)
\end{gathered}
$$

where $q(t) \in R^{n}, u(t) \in R^{r}, y(t) \in R^{m}$, are vectors of the state, input, and output variables, $A \in R^{n \times n}, C \in R^{m \times n}$ are real finite values matrices, $m, r<n$ and $\mathrm{h}_{i}(\theta(t))$ is averaging weight for the $i$-th rule, representing the normalized grade of fuzzy membership (membership function). By definition, the membership functions satisfy the convex sum properties

$$
0 \leq \mathrm{h}_{i}(\theta(t)) \leq 1, \quad \sum_{i=1}^{s} \mathrm{~h}_{i}(\theta(t))=1 \quad \text { for all } i \in\langle 1, \ldots, s\rangle
$$

where $s$ is the number of linear models (fuzzy rules) and

$$
\theta(t)=\left[\begin{array}{llll}
\theta_{1}(t) & \theta_{2}(t) & \mathrm{L} & \theta_{p}(t)
\end{array}\right]
$$

is $p$ dimensional vector of the premise variables. It is assumed that the premise variable is a system state variable, or a measurable external variable, while none of the premise variables does not depend on any element of the input variables vector $u(t)$. In the above sense, the fuzzy model of a system can be interpreted as a combination of $s$ linear models through the set of membership functions $\left\{h_{i}(\theta(t)), i=1,2, \ldots, s\right\}$. More details can be found, e.g., in [6], [12].

It is supposed that the couples $\left(A_{i}, C\right)$ are observable for all $i=1,2, \ldots, s$, as well as the matrix $C$ occurs in all local models and the number of input variables $r$ is equal to the number of output variables $m$ (the dynamic system is a square system). 


\section{TAKAGI-SUGENO FUZZY OBSERVER DESIGN}

The conventional fuzzy observer can be constructed as follows

$$
\begin{gathered}
\dot{\boldsymbol{q}}_{e}(t)=\sum_{i=1}^{s} h_{i}(\boldsymbol{\theta}(t))\left(\boldsymbol{A}_{i} \boldsymbol{q}_{e}(t)+\boldsymbol{B}_{i} \boldsymbol{u}(t)+\boldsymbol{J}_{i}\left(\boldsymbol{y}(t)-\boldsymbol{y}_{e}(t)\right)\right. \\
y_{e}(t)=C q_{e}(t)
\end{gathered}
$$

where $q_{e}(t) \in R^{n}$ is estimation of the system state vector (the fuzzy observer state vector) and $J_{i} \in R^{n \times m}, i=1,2, \ldots, s$ is the set of the observer gain matrices.

\section{Lemma 1}

The fuzzy observer (5), (6) is stable if there exist a positive definite symmetric matrix $P \in R^{n \times n}$ and matrices $Y_{i} \in R^{n \times m}$ such that for all $i=1,2, \ldots, s$

$$
\begin{gathered}
P=P^{T}>0 \\
P A_{i}+A_{i}^{T} P-Y_{i} C-C^{T} Y_{i}^{T}<0
\end{gathered}
$$

When the above conditions hold, i.e. if $Y_{i}$ and the non-singular matrix $P$ are solutions of (7), (8), the set of the observer gain matrices $J_{i}$ is given by the following equations

$$
J_{i}=P^{-1} Y_{i}
$$

Proof: (compare, e.g., [11]) Introducing the error between the system state vector and the observer state vector as follows

$$
e(t)=q(t)-q_{e}(t)
$$

and performing the time derivative of the error $e(t)$, then exploiting (1) and (10) it is

$$
\dot{e}(t)=\dot{\boldsymbol{q}}(t)-\dot{\boldsymbol{q}}_{e}(t)=\sum_{i=1}^{s} h_{i}(\boldsymbol{\theta}(t))\left(\boldsymbol{A}_{i}\left(\boldsymbol{q}(t)-\boldsymbol{q}_{e}(t)\right)-\boldsymbol{J}_{i}\left(\boldsymbol{y}(t)-\boldsymbol{y}_{e}(t)\right)\right)
$$

which can be written using (2), (11) as follows

$$
\dot{e}(t)=\sum_{i=1}^{s} h_{i}(\boldsymbol{\theta}(t)) \boldsymbol{A}_{e i} \boldsymbol{e}(t)
$$

where

$$
A_{e i}=A_{i}-J_{i} C
$$

Defining the Lyapunov function of the form

$$
v(e(t))=e^{T}(t) P e(t)>0
$$

where $P=P^{T}>0$, then evaluating the time derivative of (14) it yields 


$$
\dot{v}(e(t))=\dot{e}^{T}(t) \boldsymbol{P e}(t)+e^{T}(t) \boldsymbol{P} \dot{e}(t)
$$

Substituting (12), (13) into (15) gives

$$
\begin{gathered}
\dot{v}(e(t))=e^{T}(t) \sum_{i=1}^{s} h_{i}(\boldsymbol{\theta}(t))\left(\boldsymbol{P}\left(\boldsymbol{A}_{i}-\boldsymbol{J}_{i} \boldsymbol{C}\right)+\left(\boldsymbol{A}_{i}-\boldsymbol{J}_{i} \boldsymbol{C}\right)^{T} \boldsymbol{P}\right) e(t) \\
P\left(A_{i}-J_{i} C\right)+\left(A_{i}-J_{i} C\right)^{T} P<0 \text { for all } i
\end{gathered}
$$

respectively.

Therefore, setting

$$
P J_{i}=Y_{i}
$$

(17) implies (8). This concludes the proof.

Considering the affine properties of the TS fuzzy models, to reduce the conservatism in solution the enhanced design criterion can be derived by using two slack matrices.

\section{Theorem 1}

The fuzzy observer (5), (6) is stable if for given positive scalar $\delta \in R$ there exist a symmetric positive definite matrix $P \in R^{n \times n}$ and matrices $S_{3} \in R^{n \times n}, Y_{i} \in R^{n \times m}$ such that for all $i=1,2, \ldots, s$

$$
\begin{gathered}
P=P^{T}>0 \\
{\left[\begin{array}{cc}
A_{i}^{T} S_{3}+S_{3}^{T} A_{i}-Y_{i} C-C^{T} Y_{i}^{T} & * \\
P-S_{3}+\delta S_{3}^{T} A_{i}-\delta Y_{i C} & -\delta\left(S_{3}+S_{3}^{T}\right)<0
\end{array}\right]<0}
\end{gathered}
$$

When the above conditions hold, the set of the observer gain matrices $J_{i}$ is given by the equations

$$
J_{i}=\left(S_{3}^{T}\right)^{-1} Y_{i}
$$

Here and hereafter $*$ denotes the symmetric item in a symmetric matrix.

Proof : Since the property of (3) and (12) asserts that

$$
\sum_{i=1}^{s} h_{i}(\boldsymbol{\theta}(t))\left(\boldsymbol{A}_{e i} \boldsymbol{e}(t)-\dot{e}(t)\right)=\mathbf{0}
$$

using arbitrary square slack matrices $S_{3}, S_{4} \in R^{n \times n}$ it yields

$$
\left(\boldsymbol{q}^{T}(t) \boldsymbol{S}_{3}^{T}+\dot{\boldsymbol{q}}^{T}(t) \boldsymbol{S}_{4}^{T}\right) \sum_{i=1}^{s} h_{i}(\boldsymbol{\theta}(t))\left(\boldsymbol{A}_{e i} \boldsymbol{e}(t)-\dot{e}(t)\right)=0
$$

Adding (23) and transposition of (23) to (15) gives 


$$
\begin{gathered}
\dot{v}(e(t))=\dot{e}^{T}(t) \boldsymbol{P e}(t)+\boldsymbol{e}^{T}(t) \boldsymbol{P} \dot{e}(t)+ \\
+\left(\boldsymbol{e}^{T}(t) \boldsymbol{S}_{3}^{T}+\dot{e}^{T}(t) \boldsymbol{S}_{4}^{T}\right) \sum_{i=1}^{s} h_{i}(\boldsymbol{\theta}(t))\left(\boldsymbol{A}_{e i} \boldsymbol{e}(t)-\dot{e}(t)\right)+ \\
+\sum_{i=1}^{s} h_{i}(\boldsymbol{\theta}(t))\left(\boldsymbol{e}^{T}(t) \boldsymbol{A}_{e i}^{T}-\dot{\boldsymbol{e}}^{T}(t)\right)\left(\boldsymbol{S}_{3} \boldsymbol{e}(t)+\boldsymbol{S}_{4} \dot{\boldsymbol{e}}(t)\right)<0
\end{gathered}
$$

Then, introducing the notation

$$
e^{o T}(t)=\left[\begin{array}{ll}
e^{T}(t) & \dot{e}^{T}(t)
\end{array}\right]
$$

after straightforward computation it can be obtained

$$
\dot{v}(\boldsymbol{q}(t))=\sum_{i=1}^{s} \mathrm{~h}_{i}(\boldsymbol{\theta}(t)) \boldsymbol{e}^{\circ T}(t) \boldsymbol{Q}_{i}^{\circ} \boldsymbol{e}^{\circ}(t)<0
$$

where

$$
\begin{gathered}
Q_{i}^{\circ}=\left[\begin{array}{cc}
\left(A_{i}-J_{i} C\right)^{T} S_{3}+S_{3}^{T}\left(A_{i}-J_{i} C\right) & P-S_{3}^{T}+\left(A_{i}-J_{i} C\right)^{T} S_{4} \\
P-S_{3}+S_{4}^{T}\left(A_{i}-J_{i} C\right) & -S_{4}-S_{4}^{T}
\end{array}\right]<0 \\
S_{4}=\delta S_{3}, \quad Y_{i}=S_{3}^{T} J_{i}
\end{gathered}
$$

where $\delta>0, \delta \in R$, then (28) implies (20). This concludes the proof.

The importance of Theorem 1 is that the Lyapunov matrix $P$ is separated from the system matrices $A_{i}, C$, i.e. there are no terms containing product of $P$ and any of them. This enables to derive design conditions with respect to natural affine properties of TS models.

It is evident, that Theorem 1 can be simple reformulated considering a symmetric matrix $S_{3}=S_{3}^{T}$.

\section{DESCRIPTOR PRINCIPLE BASED DESIGN METHOD}

The results given by Theorem 1 can be generalized using descriptor principle and are formulated as the following theorem.

\section{Theorem 2}

The fuzzy observer (22), (23) is stable if for given positive scalar $\delta \in R$ there exist a symmetric positive definite matrix $P_{1} \in R^{n \times n}$ and matrices $P_{2}, P_{3} \in R^{n \times n}, \quad Y_{i} \in R^{n \times m}$ such that for all $i=1,2, \ldots, s$

$$
\begin{gathered}
P_{1}=P_{1}^{T}>0, \\
P_{2}+P_{2}^{T}>0 \\
{\left[\begin{array}{cc}
A_{i}^{T} P_{3}+P_{3}^{T} A_{i}-Y_{i} C-C^{T} Y_{i}^{T} & * \\
P_{1}-P_{3}+\delta P_{3}^{T} A_{i}-\delta Y_{i} C & P_{2}+P_{2}^{T}-\delta\left(P_{3}+P_{3}^{T}\right)
\end{array}\right]<0}
\end{gathered}
$$

When the above conditions hold, the set of the observer gain matrices $J_{i}$ is given by the set of the equations

$$
J_{i}=\left(P_{3}^{T}\right)^{-1} Y_{i}
$$

Proof : Using the equality (22), then with the identities 


$$
\dot{e}(t)=\dot{e}(t), \quad \mathbf{0}=\mathbf{0}
$$

an equivalent form of (22)can be written as

$$
\left[\begin{array}{cc}
\boldsymbol{I}_{n} & \mathbf{0} \\
\mathbf{0} & \mathbf{0}
\end{array}\right]\left[\begin{array}{l}
\dot{e}(t) \\
\ddot{e}(t)
\end{array}\right]=\left[\begin{array}{c}
\dot{e}(t) \\
\mathbf{0}
\end{array}\right]=\sum_{i=1}^{s} h_{i}(\boldsymbol{\theta}(t))\left[\begin{array}{cc}
\mathbf{0} & \boldsymbol{I}_{n} \\
\boldsymbol{A}_{e i} & -\boldsymbol{I}_{n}
\end{array}\right]\left[\begin{array}{c}
e(t) \\
\dot{e}(t)
\end{array}\right]
$$

or more generally

$$
\boldsymbol{E}^{\circ} \dot{\boldsymbol{e}}^{\circ}(t)=\sum_{i=1}^{s} h_{i}(\boldsymbol{\theta}(t)) \boldsymbol{A}_{e i}^{\circ} e^{\circ}(t)
$$

where $e^{\circ}(t)$ is given in (25) and

$$
E^{\circ}=E^{\circ} T=\left[\begin{array}{cc}
I_{n} & 0 \\
0 & 0
\end{array}\right], \quad A_{e i}^{\circ}=\left[\begin{array}{cc}
0 & I_{n} \\
A_{e i} & -I_{n}
\end{array}\right]
$$

Defining the Lyapunov function of the form

$$
v\left(e^{\circ}(t)\right)=e^{\circ T}(t) E^{\circ T} P^{\circ} e^{\circ}(t)>0
$$

where

$$
E^{\circ T} P^{\circ}=P^{\circ T} E^{\circ} \geq 0
$$

then the derivative of (36) becomes

$$
\dot{v}\left(e^{\circ}(t)\right)=\dot{e}^{\circ T}(t) \boldsymbol{E}^{\circ T} \boldsymbol{P}^{\circ} e^{\circ}(t)+\boldsymbol{e}^{\circ T}(t) \boldsymbol{P}^{\circ T} \boldsymbol{E}^{\circ} \dot{\boldsymbol{e}}^{\circ}(t)<0
$$

Inserting (34) in (38) it yields

$$
\begin{gathered}
\dot{v}\left(e^{\circ}(t)\right)=e^{\circ T}(t) \sum_{i=1}^{s} h_{i}(\boldsymbol{\theta}(t))\left(\boldsymbol{P}^{\circ T} \boldsymbol{A}_{e i}^{\circ}+\boldsymbol{A}_{e i}^{\circ T} \boldsymbol{P}^{\circ}\right) \boldsymbol{e}^{\circ}(t)<0 \\
P^{\circ T} A_{e i}^{\circ}+A_{e i}^{\circ T} P^{\circ}<0 \text { forall } i
\end{gathered}
$$

respectively. Defining

$$
P^{\circ}=\left[\begin{array}{ll}
P_{1} & P_{2} \\
P_{3} & P_{4}
\end{array}\right]
$$

then (35), (37) implies

$$
P_{1}=P_{1}^{T}>0
$$

and using (35) within (13) in (40) it yields

$$
\left[\begin{array}{cc}
0 & \left(A_{i}-J_{i} C\right)^{T} \\
I_{n} & -I_{n}
\end{array}\right]\left[\begin{array}{ll}
P_{1} & P_{2} \\
P_{3} & P_{4}
\end{array}\right]+\left[\begin{array}{ll}
P_{1}^{T} & P_{3}^{T} \\
P_{2}^{T} & P_{4}^{T}
\end{array}\right]\left[\begin{array}{cc}
0 & I_{n} \\
A_{i}-J_{i} C & -I_{n}
\end{array}\right]<0
$$

After some algebraic manipulations (43) takes the following form

$$
\left[\begin{array}{cc}
\left(A_{i}-J_{i} C\right)^{T} P_{3}+P_{3}^{T}\left(A_{i}-J_{i} C\right) & \left(A_{i}-J_{i} C\right)^{T} P_{4}+P_{1}^{T}-P_{3}^{T} \\
P_{1}-P_{3}+P_{4}^{T}\left(A_{i}-J_{i} C\right) & P_{2}+P_{2}^{T}-P_{4}-P_{4}^{T}
\end{array}\right]<0
$$


Setting

$$
P_{4}=\delta P_{3}, \quad Y_{i}=P_{3}^{T} J_{i}
$$

where $\delta>0, \delta \in R$, then (44) implies (30). This concludes the proof.

\section{Remark 1}

It is naturally to point out that Theorem 2 is an extension and generalization of Theorem 1 , since setting

$$
P_{2}=0, \quad P_{1}=P, \quad P_{3}=S_{3}
$$

(29), (30) implies (19), (20), respectively. The extension of (30) reflects the Krasovskii theorem property [13] allowing either to consider (24) in the following form

$$
\begin{gathered}
\dot{v}(e(t))=\dot{e}^{T}(t) P e(t)+e^{T}(t) P \dot{e}(t)+ \\
+\left(e^{T}(t) \boldsymbol{S}_{3}^{T}+\dot{e}^{T}(t) \boldsymbol{S}_{4}^{T}\right) \sum_{i=1}^{s} h_{i}(\boldsymbol{\theta}(t))\left(\boldsymbol{A}_{e i} e(t)-\dot{e}(t)\right)+ \\
+\sum_{i=1}^{s} h_{i}(\boldsymbol{\theta}(t))\left(e^{T}(t) \boldsymbol{A}_{e i}^{T}-\dot{e}^{T}(t)\right)\left(\boldsymbol{S}_{3} e(t)+\boldsymbol{S}_{4} \dot{e}(t)\right)<-\dot{e}^{T}(t)\left(\boldsymbol{S}_{2}+\boldsymbol{S}_{2}^{T}\right) \dot{e}(t)<0
\end{gathered}
$$

or, equivalently, to define the Lyapunov function in the proof of Theorem 2 as follows

$$
v(e(t))=e^{T}(t) \boldsymbol{P e}(t)+\int_{0}^{t} \dot{e}^{T}(\tau)\left(\boldsymbol{S}_{2}+\boldsymbol{S}_{2}^{T}\right) \dot{e}(\tau) d \tau>0
$$

and, as initially, to apply (24) in the proof and, finally, to compare the obtained result with (29), (30) setting

$$
P=P_{1}, \quad S_{3}=P_{3}, \quad S_{2}=P_{2}
$$

\section{Corollary 1}

Considering

$$
P_{2}=0, \quad P_{4}=0, \quad P_{1}=P_{3}
$$

then (44) reduces to

$$
\left[\begin{array}{cc}
\left(A_{i}-J_{i} C\right)^{T} P_{1}+P_{1}^{T}\left(A_{i}-J_{i} C\right) & 0 \\
0 & 0
\end{array}\right] \leq 0
$$

which implies

$$
\left(A_{i}-J_{i} C\right)^{T} P_{1}+P_{1}^{T}\left(A_{i}-J_{i} C\right)<0
$$

It is obvious that with

$$
P=P_{1}=P_{1}^{T}, \quad Y_{i}=P_{1}^{T} J_{i}=P J_{i}
$$

(52) implies (8).

These modifications give the possibility to achieve the degree of conservatism that is most appropriate for a TS system. 


\section{ILLUSTRATIVE EXAMPLE}

The considered system is represented by the TS fuzzy model (1), (2) with $\mathrm{s}=3$ and the system model parameters

$$
\begin{gathered}
A_{1}=\left[\begin{array}{rrr}
-1.0522 & -1.8666 & 0.5102 \\
-0.4380 & -5.4335 & 0.9205 \\
-0.5522 & 0.1334 & -0.4898
\end{array}\right], A_{2}=\left[\begin{array}{rrr}
-1.0565 & -1.8661 & 0.5116 \\
-0.4380 & -5.4359 & 0.9214 \\
-0.5565 & 0.1339 & -0.4884
\end{array}\right] \\
A_{3}=\left[\begin{array}{rrr}
-1.0602 & -1.8657 & 0.5133 \\
-0.4381 & -5.4353 & 0.9216 \\
-0.5602 & 0.1343 & -0.4867
\end{array}\right], B=\left[\begin{array}{rr}
3 & 1 \\
1 & -1 \\
3 & 0
\end{array}\right], C^{T}=\left[\begin{array}{rr}
1 & 0 \\
1 & 1 \\
0 & 1
\end{array}\right]
\end{gathered}
$$

where matrices $B_{i}$ are the same for all $i$ and the premise variable and the membership functions for approximation of $f\left(q_{1}(t)\right)$ in the prescribed sector are given as

$$
\begin{gathered}
\theta(t)=\left[\begin{array}{c}
\theta_{1}(t) \\
\theta_{2}(t) \\
\theta_{3}(t)
\end{array}\right], \theta_{i}=\left\{\begin{array}{c}
\theta_{1}(t) \text { if } q_{1}(t) \text { is about } 5, \\
\left\{\theta_{2}(t) \text { if } q_{1}(t) \text { is about } 0,\right. \\
\theta_{3}(t) \text { if } q_{1}(t) \text { is about }-5,
\end{array}\right. \\
h_{1}\left(\theta_{2}(t)\right)=1-\frac{1}{5}\left|\theta_{2}(t)-5\right|, \quad h_{2}\left(\theta_{1}(t)\right)=1-\frac{1}{5}\left|\theta_{1}(t)\right|, \quad h_{3}\left(\theta_{3}(t)\right)=1-\frac{1}{5}\left|\theta_{3}(t)+5\right|
\end{gathered}
$$

Solving the variables $P, Y_{i}, i=1,2,3$ satisfying (7), (8) via the LMI technique using toolbox SeDuMi [14] gave the following results

$$
\begin{gathered}
P=\left[\begin{array}{rrr}
0.6353 & -0.1200 & -0.0377 \\
-0.1200 & 0.2368 & 0.0521 \\
-0.0377 & 0.0521 & 0.6776
\end{array}\right] \\
Y_{1}=\left[\begin{array}{rr}
-0.0969 & -0.1124 \\
-0.3185 & -0.1973 \\
0.0101 & 0.1690
\end{array}\right], Y_{2}=\left[\begin{array}{rr}
-0.0992 & -0.1120 \\
-0.3173 & -0.1979 \\
0.0089 & 0.1698
\end{array}\right], Y_{3}=\left[\begin{array}{rr}
-0.1002 & -0.1118 \\
-0.3170 & -0.1977 \\
0.0082 & 0.1709
\end{array}\right]
\end{gathered}
$$

and, besides, the fuzzy observer gain matrices were obtained as follows

$$
J_{1}=\left[\begin{array}{rr}
-0.4474 & -0.3634 \\
-1.5966 & -1.0859 \\
0.1126 & 0.3127
\end{array}\right], J_{2}=\left[\begin{array}{rr}
-0.4502 & -0.3632 \\
-1.5925 & -1.0888 \\
0.1104 & 0.3140
\end{array}\right], J_{3}=\left[\begin{array}{rr}
-0.4517 & -0.3627 \\
-1.5914 & -1.0881 \\
0.1092 & 0.3157
\end{array}\right]
$$

guaranteeing the stable eigenvalues spectra of the local observer system matrices in such a way that

$$
\begin{gathered}
\rho\left(A_{e 1}\right)=\{-0.7560,-1.7011 \pm 1.1316 \mathrm{i}\}, \quad \rho\left(A_{e 2}\right)=\{-0.7575,-1.7029 \pm 1.1269 \mathrm{i}\} \\
\rho\left(A_{e 3}\right)=\{-0.7599,-1.7034 \pm 1.1265 \mathrm{i}\}
\end{gathered}
$$

Applying the same toolbox to solve LMIs (19), (20) conditioned by $\delta=1$, the obtained set of matrix variables was as follows 


$$
\begin{gathered}
P=\left[\begin{array}{rrr}
0.6415 & -0.1152 & 0.0157 \\
-0.1152 & 0.7158 & -0.0994 \\
0.0157 & -0.0994 & 0.7004
\end{array}\right], S_{3}=\left[\begin{array}{rrr}
0.3577 & -0.0386 & -0.0348 \\
-0.1063 & 0.1468 & 0.0100 \\
0.0595 & 0.0316 & 0.3587
\end{array}\right] \\
Y_{1}=\left[\begin{array}{rr}
-0.0188 & 0.0224 \\
-0.1142 & -0.0324 \\
-0.1452 & 0.1856
\end{array}\right], Y_{2}=\left[\begin{array}{rr}
-0.0191 & 0.0248 \\
-0.1155 & -0.0323 \\
-0.1465 & 0.1828
\end{array}\right], Y_{3}=\left[\begin{array}{rr}
-0.0227 & 0.0237 \\
-0.1138 & -0.0327 \\
-0.1458 & 0.1833
\end{array}\right]
\end{gathered}
$$

so that the local observers gain matrices were given as

$$
J_{1}=\left[\begin{array}{rr}
-0.2067 & -0.1318 \\
-0.7449 & -0.3663 \\
-0.4039 & 0.5149
\end{array}\right], J_{2}=\left[\begin{array}{rr}
-0.2097 & -0.1226 \\
-0.7543 & -0.3616 \\
-0.4076 & 0.5079
\end{array}\right], J_{3}=\left[\begin{array}{rr}
-0.2171 & -0.1270 \\
-0.7449 & -0.3652 \\
-0.4067 & 0.5088
\end{array}\right]
$$

This set of gains embedded the eigenvalues spectra of the local observer system matrices as follows

$$
\begin{gathered}
\rho\left(A_{e 1}\right)=\{-4.1633,-1.0047 \pm 0.0879 \mathrm{i}\}, \quad \rho\left(A_{e 2}\right)=\{-4.1600,-1.0016 \pm 0.0804 \mathrm{i}\} \\
\rho\left(A_{e 3}\right)=\{-4.1703,-0.9967 \pm 0.0917 \mathrm{i}\}
\end{gathered}
$$

Finally, solving LMIs (29), (30) conditioned by $\delta=1$, a feasible solution produced the following LMI variables

$$
\begin{gathered}
P_{1}=\left[\begin{array}{rrr}
1.0236 & -0.2562 & 0.0263 \\
-0.2562 & 1.0633 & -0.1593 \\
0.0263 & -0.1593 & 1.1439
\end{array}\right], P_{3}=\left[\begin{array}{rrr}
0.7114 & -0.1530 & 0.1444 \\
-0.1842 & 0.2562 & -0.0228 \\
-0.1177 & 0.1345 & 0.7273
\end{array}\right] \\
Y_{1}=\left[\begin{array}{rr}
-0.1798 & 0.0085 \\
-0.1937 & -0.0745 \\
-0.3158 & 0.1619
\end{array}\right], Y_{2}=\left[\begin{array}{rr}
-0.1816 & 0.0096 \\
-0.1938 & -0.0745 \\
-0.3186 & 0.1637
\end{array}\right], Y_{3}=\left[\begin{array}{rr}
-0.1833 & 0.0106 \\
-0.1937 & -0.0744 \\
-0.3210 & 0.1655
\end{array}\right]
\end{gathered}
$$

where, for simplicity, $P_{2}$ is not listed. This result provides TS fuzzy state observer with the following local gain matrices

$$
J_{1}=\left[\begin{array}{rr}
-0.5430 & -0.0672 \\
-0.8942 & -0.4476 \\
-0.3544 & 0.2220
\end{array}\right], J_{2}=\left[\begin{array}{rr}
-0.5462 & -0.0653 \\
-0.8950 & -0.4475 \\
-0.3576 & 0.2241
\end{array}\right], J_{3}=\left[\begin{array}{rr}
-0.5491 & -0.0635 \\
-0.8951 & -0.4470 \\
-0.3604 & 0.2262
\end{array}\right]
$$

and with the eigenvalues spectra of the local observer system matrices

$$
\begin{gathered}
\rho\left(A_{e 1}\right)=\{-4.0034,-0.6547 \pm 0.1088 \mathrm{i}\}, \quad \rho\left(A_{e 2}\right)=\{-4.0056,-0.6553 \pm 0.1090 \mathrm{i}\} \\
\rho\left(A_{e 3}\right)=\{-4.0060,-0.6557 \pm 0.1095 \mathrm{i}\}
\end{gathered}
$$

Applying the designed to the TS fuzzy system model with the initial condition

$$
q_{e}^{T}(0)=0, \quad u^{T}(t)=0, \quad q^{T}(0)=\left[\begin{array}{lll}
0.3 & 0.6 & 0.9
\end{array}\right]
$$


the simulation results are stated in Fig. 1 to Fig. 3 to illustrate the estimated output behaviour of the system sequentially as the observers parameters were computed using Lemma 1 , Theorem 1 and Theorem 2. It is evident that the best compromise in the settling time and overshooting gives the result of Theorem 2 .

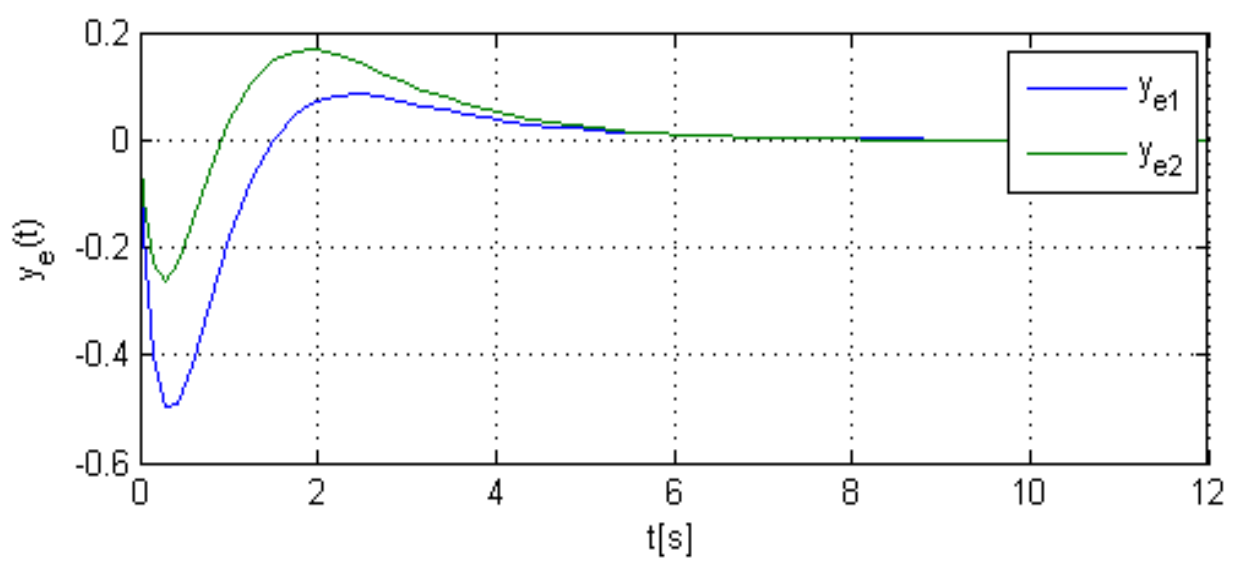

Figure 1: TS fuzzy observer output variables response (based on Lemma 1 results)

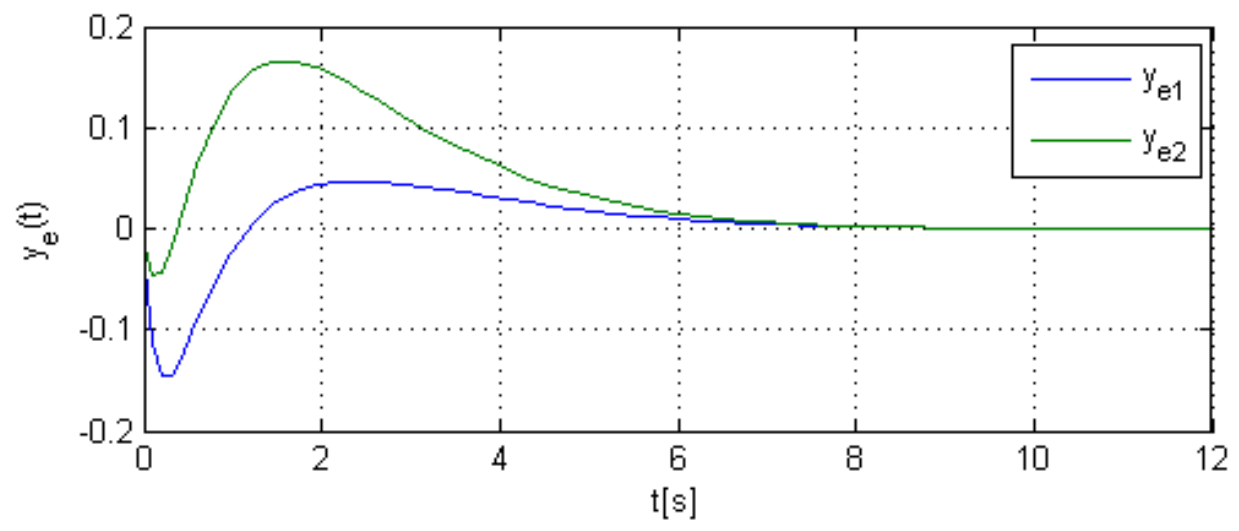

Figure 2: TS fuzzy observer output variables response (based on Theorem 1 results)

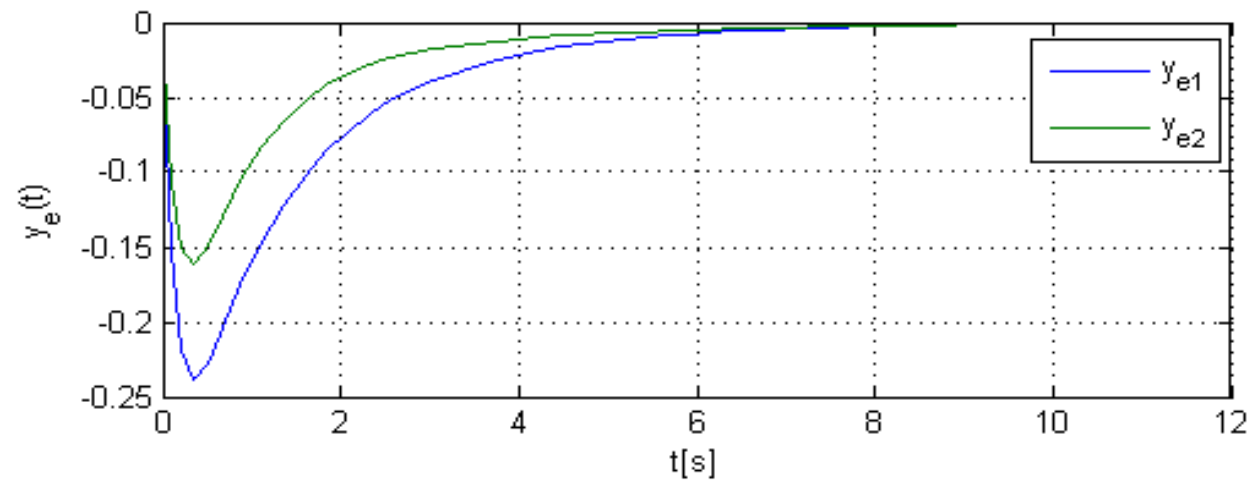

Figure 3: TS fuzzy observer output variables response (based on Lemma 2 results) 


\section{CONCLUDING REMARKS}

New approach for output dynamic feedback control design is presented in this paper. By the proposed procedure the control problem is parameterized in such LMIs set with one additional LME which admit more freedom in guaranteeing the output feedback control performance for a bi-proper dynamic controller and by LMIs set only for a strictly proper dynamic output controller. Sufficient conditions of the controller existence manipulating the stability of the closed-loop systems imply the control structure, which stabilize the system in the sense of Lyapunov and the controller design tasks is a solvable numerical problem. An additional benefit of the method is that controller uses minimum feedback information with respect to desired system output and the approach is enough flexible to allow the inclusion of additional design condition bounds.

\section{ACKNOWLEDGEMENTS}

The work presented in the paper was supported by VEGA, the Grant Agency of the Ministry of Education and the Academy of Science of Slovak Republic, under Grant No. 1/0348/14. This support is very gratefully acknowledged.

\section{REFERENCES}

[1] Thau, F.E. (1973) "Observing the state of nonlinear dynamical systems", International Journal of Control, Vol. 17, No. 5, pp. 471-479.

[2] Koshkouei, A.J. \& Zinober, A.S.I. (1999) "Partial Lipschitz nonlinear sliding mode observers", Proceedings of the 7th Mediterranean Conference on Control and Automation MED99, Haifa, Israel, pp. 2350-2359.

[3] Takagi, T. \& Sugeno, M. (1985) "Fuzzy identification of systems and its applications to model-ling and control", IEEE Transactions on Systems, Man, and Cybernetics, Vol. 15, No. 1, pp. 116-132.

[4] Ichalal, D., Marx, B., Ragot, J. \& Maquin, D. (2007) "Design of observers for Takagi-Sugeno discretetime systems with unmeasurable premise variables", Proceedings of the 5th Workshop on Advanced Control and Diagnosis ACD 2007, Grenoble, France.

[5] Gao, Z., Shi, X. \& Ding, S.X. (2008) "Fuzzy state/disturbance observer design for T-S fuzzy systems with application to sensor fault estimation", IEEE Transactions on Systems, Man, and Cybernetics, Part B: Cybernetics, Vol. 38, No. 3. pp. 875-880.

[6] Tanaka, K. \& Wang, H.O. (2001) Fuzzy Control Systems Design and Analysis: A Linear Matrix Inequality Approach. New York, John Wiley \& Sons.

[7] Lu, G., Ho, D.W.C. \& Zheng, Y. (2004) "Observers for a class of descriptor systems with Lip-schitz constraint", Proceeding of the 2004 American Control Conference, Boston, MA, USA, pp. 3474-3479.

[8] Fridman, E. \& Shaked. U. (2002) "A descriptor system approach to Hळ control of linear time-delay systems", IEEE Transactions on Automatic Control, Vol. 47, No. 2, pp. 253-270.

[9] Ilhem, K., Dalel, J., Saloua, B.H.A. \& Naceur, A.M. (2012) "Observer design for Takagi-Sugeno descriptor system with Lipschitz constraints", International Journal of Instrumentation and Con-trol Systems. Vol. 2, No. 2, pp. 13-25.

[10] Tong, S., Yang, G. \& Zhang, W. (2011) "Observer-based fault-tolerant control against sensor failures for fuzzy systems with time delays", International Journal of Applied Mathematics and Computer Science. Vol. 21, No. 4, pp. 617-627.

[11] Filasova, A. \& Krokavec, D. (2013) "On the Takagi-Sugeno model-based state estimation for one class of bilinear systems", Proceedings of the 14th International Carpathian Control Confe-rence ICCC'13, Rytro, Poland, pp. 83-87.

[12] Krokavec, D. \& Filasova, A. (2012) "Optimal fuzzy control for a class of nonlinear systems", Mathematical Problems in Engineering, Vol. 2012, ID 481942, 29 p.

[13] Haddad, W.M. \& Chellaboina, V. (2008) Nonlinear Dynamical Systems and Control: A Lyapu-nov Based Approach, Princeton, NJ, USA, Princeton University Press.

[14] Peaucelle, D., Henrion, D., Labit, Y. \& Taitz, K. (2002) User's Guide for SeDuMi Interface 1.04, Toulouse, France, LAAS-CNRS. 


\section{AUTHORS}

Anna Filasova graduated in technical cybernetics and received M.Sc. degree in 1975, and Ph.D. degree in 1993 both from the Faculty of Electrical Engineering and Informatics, Technical University of Kosice, Slovakia. In 1999 she was appointed Associated Professor from the Technical University in Kosice in technical cybernetics. She is with the Department of Cybernetics and Artificial Intelligence, Faculty of Electrical Engineering and Informatics, Technical University of Kosice, and she has been working with as an Assistant Professor from 1975 to 1999. Her main research interests are in robust and predictive control, decentralized control, large-scale system optimization, and control reconfiguration.

Dusan Krokavec received M.Sc. degree in automatic control in 1967 and Ph.D. degree in technical cybernetics in 1982 from the Faculty of Electrical Engineering, Slovak University of Technology in Bratislava, Slovakia. In 1984 he was promoted Associated Professor from the Technical University in Kosice, Slovakia, and in 1999 he was appointed Full Professor in automation and control. He is with the Department of Cybernetics and Artificial Intelligence, Faculty of Electrical Engineering and Informatics, Technical University of Kosice. In the long term, he specializes in stochastic processes in dynamic systems, digital control systems and digital signal processing, and in dynamic system fault diagnosis. 